\title{
Locally Adjusted Cubic-Spline Capping for Reconstructing Seasonal Trajectories of a Satellite-Derived Surface Parameter
}

\author{
Jing M. Chen, Feng Deng, and Mingzhen Chen
}

\begin{abstract}
Satellite-derived vegetation indices and their resulting surface parameters, such as the leaf area index (LAI), are inevitably affected by the atmosphere. Errors in the atmospheric corrections can often be easily identified in a seasonal trajectory of a surface parameter because the atmospheric effect generally causes erratic reductions in vegetation indices. A locally adjusted cubic-spline capping (LACC) method is developed here to screen affected data points in a pixel and to replace them through temporal interpolation. In LACC, a variable local smoothing parameter, which controls the local smoothness of the fitted curve, is automatically determined according to the local curvature of the original seasonal variation pattern. An iteration procedure is designed to produce a seasonal capping curve by progressively replacing abnormally low values with fitted values. This method has two advantages over existing methods based on harmonics, namely: 1) cubic splines are flexible for simulating a wide range of seasonal variation patterns and 2) a variable local smoothing parameter allows the fitted capping curve to mimic either rapid or slow variation patterns in various seasons. The capping curve is also mathematically differentiable for further applications. The effectiveness of this method is demonstrated through case studies for several cover types in China and processing a series of Moderate Resolution Imaging Spectroradiometer LAI images of China in 2001.
\end{abstract}

Index Terms-Cubic spline, leaf area index (LAI), residual cloud screening, seasonal trajectory.

\section{INTRODUCTION}

$\mathbf{T}$ HE LEAF area index (LAI), defined as one-half the total green leaf area (all sided) per unit ground surface area [1], is one of the surface parameters that can be derived using satellite optical imagery and is a basic input to many regional and global water and carbon cycle models. Global LAI products are now routinely produced from data acquired by Moderate Resolution Imaging Spectroradiometer (MODIS) sensors onboard Terra and Aqua platforms as part of the National Aeronautics and Space Administration (NASA) Earth

Manuscript received June 9, 2004; revised November 29, 2005. This work was supported in part by the Canadian International Development Agency and in part by the European Space Agency GLOBCARBON Project.

J. M. Chen is with the Department of Geography and Program in Planning, University of Toronto, Toronto, ON M5S 3G3, Canada and also with York University, Toronto, ON M3J 1P3, Canada (e-mail: chenj@geog.utoronto.ca).

F. Deng is with the Department of Geography and Program in Planning, University of Toronto, Toronto, ON M5S 3G3, Canada.

M. Chen is with the Department of Geography and Program in Planning, University of Toronto, Toronto, ON M5S 3G3, Canada and also with Auburn University, Auburn, AL 36849 USA.

Digital Object Identifier 10.1109/TGRS.2006.872089
Observation System [12]. Other LAI products were also produced using imagery from the Advanced Very High Resolution Radiometer (AVHRR), VEGETATION [2], [5], and the Multiangle Imaging SpectroRadiometer [8]. The European Space Agency also funded a new initiative named GLOBCARBON to produce global LAI products using the VEGETATION, Along Track Scanning Radiometer, and Medium Resolution Imaging Spectrometer sensors [13] for the purpose of global carbon cycle modeling. These new data sets provided unprecedented opportunities for studying the global ecology and the impact on the Earth's climate. Since its inception in 2000, the MODIS LAI product (MOD15A2) has been increasingly used for various global and regional studies [10], [11]. In the present study, we address the issue of residual effects of atmospheric correction and other artifacts on the retrieved LAI based its seasonal variation pattern. The retrieval of LAI using multispectral remote sensing data depends on the reflectances in the red, near-infrared (NIR), and sometimes shortwave infrared (SWIR) bands at the surface level, which are often very sensitive to the atmospheric effects including clouds, aerosols, water vapor, ozone, etc. [21], [22]. Whereas much of these effects can be removed using real-time or near real-time atmospheric observations made by the same sensor and other sensors [9], [22], the remaining effects can sometimes be very large. These remaining effects generally cause increases in the reflectance in the red band much more than in the NIR band, resulting in decreases in the retrieved LAI. In a seasonal trajectory, these effects can be identified as abnormally low values. Seasonal variation patterns of remotely sensed surface parameters can therefore provide an additional quality control [3], [4]. Variations in other factors, such as satellite view angle and solar illumination angle, can also cause errors in retrieved LAI, although these angular effects are considered in the MODIS LAI algorithm [11]. Inaccuracies in modeling the angular variation pattern can cause either positive or negative errors in retrieved LAI [3], and excessive atmospheric corrections on the red band can also cause positive biases in vegetation indices and LAI. The method presented here is only effective in removing the negative errors.

Different techniques have been used to determine the seasonal patterns of surface parameters and to exercise a final quality control through identifying dates of erroneous data and to replace them through temporal interpolation [7], [17], [18], [23]. Based on Roerink et al. [15], Verhoef et al. [20] developed a method for reconstructing the seasonal series of the normalized difference vegetation index (NDVI) through Fourier 
analysis. Similar Fourier methods have been used in the FASIR procedure by scientists at NASA [16] and further developed in the ABC3 model by scientists at the Canada Centre for Remote Sensing (CCRS) [3], [4]. As the number of cloud-free scenes in a year is often small at a given location, these Fourier analysis methods make use of only two harmonic frequencies representing the annual and semiannual cycles. The resulting curve after combining these two harmonics is often too rigid to simulate realistic seasonal changes in surface parameters. Many natural seasonal variations, such as the rapid vegetation change at the beginning and end of the growing season, bimodal patterns in double-cropping agricultural systems, cannot be well fitted with only two harmonics. For near square-wave type of variation patterns, such as seasonal variation of LAI of deciduous forests, harmonics are very inefficient in curve fitting, needing tens of harmonics to mimic rapid changes. Viovy and Arino [23] proposed a simple method to remove atmospherically affected data through a moving-window curve capping procedure, but the method depends on a number of good observations in a given moving window, and the result is sensitive to the choice of the size of the moving window. The resulting curve is often mathematically unsmooth for making the first and second derivatives needed for determining growing season parameters, such as leaf-on and leaf-off dates. In processing regional and global remote sensing products of land surface parameters, a flexible and generally applicable curving fitting technique is yet needed for seasonal trajectory analysis. The objectives of this study are therefore as follows: 1) to develop a locally adjusted cubic spline capping (LACC) method, which can produce flexible and mathematically smooth capping curves and fit rapid seasonal changes, for the purpose of identifying atmosphere-contaminated data points and their replacement through temporal interpolation, and 2) to test the method for a region using the MODIS LAI product to demonstrate its effectiveness and discuss possible limitations. Although the application of the method is made on LAI time series, it can also be applied to other parameters such as vegetation indices and the fraction of photosynthetically active radiation (FPAR) absorbed by vegetation.

\section{PRinciples of LACC AND Globally Uniform CubiC-SPline CAPPING (GUCC)}

LACC and GUCC methods were developed here based on principles of the locally weighted regression method, or locally weighted scatterplot smoothing (LOESS), developed for curve fitting on noisy data series [6]. Different mathematical functions, e.g., polynomials of different orders, can be used in LOESS to do piecewise smooth curve fitting for a time series. In the application of LOESS, a preliminary curve is first fitted to the existing data points, and weights to all the points for the second curve fitting are determined according to the departures of the points from the preliminary curve. The final smoothed curve is found after applying the weights in the second curve fitting. The weight is the smallest for those with the largest departure, and the weight is symmetric above and below the curve. Although LOESS is capable of fitting desired curves shapes, it has two ma- jor problems when applied to remote sensing series, namely: 1) symmetric weight distribution is not suitable for seasonal trajectory applications because atmospheric effects generally cause negative biases in vegetation indices and their resulting surface parameters. Our purpose of curve fitting is to identify this type of biases and produce a seasonal capping curve. Points below the curve should therefore receive much smaller weights than those above the curve; and 2) preliminary curve fitting needs a control on the curve smoothness, and it is difficult to set a global smoothing parameter as the seasonal variability in surface parameters is generally not uniform with time. To overcome these problems, we developed the LACC method. Cubic splines are chosen for their flexibility in fitting desired curve shapes. Without a control on the curve smoothness, a cubic spline can exactly fit all data points so no departures of the data points from the fitted curve are found. A smoothing parameter is therefore used to control the smoothness of the cubic spline to identify abnormally low values. LACC differs from LOESS in the following ways: 1) after the preliminary curve fitting, all points below the fitted curve are replaced by the fitted values, whereas others remain unchanged. An iterative procedure is followed to repeat this process until an effective capping for the original data series is found. We found that three iterations are generally sufficient, so this is also computationally efficient for image processing and 2) preliminary curve is fitted with a fixed global smoothing parameter, and based on the curvature of the fitted curve, a local curve smoothness matrix is automatically constructed to scale the relative values of the local smooth parameter at all data points, and this matrix is then used for the second curve fitting and iteration. In this way, the problem with the uneven seasonal variability is minimized, e.g., at the beginning and the end of the growing season, when the curvatures are the largest, the smoothing parameter is the largest to capture the rapid changes. The GUCC method makes use of only one value of the smoothing parameter and is used here as a comparison to LACC. Mathematically, the LACC and GUCC methods are described below.

For one pixel, there can be $n+1$ cloudless (clear) remote sensing images in a year. The remotely derived surface parameter, such as LAI or NDVI, is denoted as $y$, and time (day of year or DOY) as $x$, so a two-dimensional array, i.e., $\left(x_{0}, y_{0}\right),\left(x_{1}, y_{1}\right), \ldots,\left(x_{n}, y_{n}\right)$, is constructed. In this series, the time step can be irregular. The first step is to bridge the gap between adjacent points $\left(x_{i}, y_{i}\right),\left(x_{i+1}, y_{i+1}\right)$ using cubic splines $S_{i}, i=0,1, \ldots, n-1$, so as to piece together a smooth curve allowing for the first and second derivatives.

The function $S_{i}$ can be expressed as

$$
S_{i}(x)=a_{i}\left(x-x_{i}\right)^{3}+b_{i}\left(x-x_{i}\right)^{2}+c_{i}\left(x-x_{i}\right)+d_{i}
$$

where $x$ ranges from $x_{i}$ to $x_{i+1}$ [19]. Under the assumption that the ends of a time series are left free, the conditions $S_{0}^{\prime \prime}\left(x_{0}\right)=2 b_{0}=0$ and $S_{n}^{\prime \prime}\left(x_{n}\right)=2 b_{n}=0$ are satisfied, and we then have

$$
b_{0}=b_{n}=0 .
$$

This function is fitted to the available data points piecewise but continuously to produce a smooth curve for the whole time 
series. The coefficients $a_{i}, b_{i}, c_{i}$, and $d_{i}$ can be derived by introducing intermediate values as follows:

$$
\begin{aligned}
h_{i-1} & =x_{i}-x_{i-1} \\
p_{i} & =2\left(h_{i-1}+h_{i}\right) \\
r_{i} & =\frac{3}{h_{i}} \\
f_{i} & =-\left(r_{i-1}+r_{i}\right)
\end{aligned}
$$

where $i=1,2, \ldots, n$, and solving the following systems of equations.

1) The coefficients $b_{i}(i=0,1, \ldots, n-1)$ are derived from

$$
\left(M+\mu Q^{T} \Gamma Q\right) b=Q^{T} y
$$

where

$$
\begin{aligned}
& M=\left[\begin{array}{cccccc}
p_{1} & h_{1} & 0 & \cdots & 0 & 0 \\
h_{1} & p_{2} & h_{2} & \cdots & 0 & 0 \\
0 & h_{2} & p_{3} & \cdots & 0 & 0 \\
\vdots & \vdots & \vdots & \ddots & \vdots & \vdots \\
0 & 0 & 0 & \cdots & p_{n-2} & h_{n-2} \\
0 & 0 & 0 & \cdots & h_{n-2} & p_{n-1}
\end{array}\right] \\
& b=\left[\begin{array}{c}
b_{1} \\
b_{2} \\
b_{3} \\
\vdots \\
b_{n-2} \\
b_{n-1}
\end{array}\right] \\
& Q^{T}=\left[\begin{array}{ccccccc}
r_{0} & f_{1} & r_{1} & 0 & \cdots & 0 & 0 \\
0 & r_{1} & f_{2} & r_{2} & \cdots & 0 & 0 \\
\vdots & \vdots & \vdots & \vdots & \ddots & \vdots & \vdots \\
0 & 0 & 0 & 0 & \cdots & r_{n-2} & 0 \\
0 & 0 & 0 & 0 & \cdots & f_{n-1} & r_{n-1}
\end{array}\right] \\
& y=\left[\begin{array}{c}
y_{0} \\
y_{1} \\
\vdots \\
y_{n}
\end{array}\right]
\end{aligned}
$$

where $Q^{T}$ is the transpose of Q. $\mu=(2(1-\lambda) / 3 \lambda)$, where $\lambda$ is a parameter related to the global smoothing parameter controlling the smoothness of the fitted curve [14]. The $\lambda$ value falls in the range from 0 to 1 . The smaller the $\lambda$ value, the smoother is the fitted curve. If the maximum value of 1 is chosen, the fitted curve would mimic the original data points, and it becomes impossible to identify problematic data points even in a noisy seasonal time series. If the value is too small, the curve becomes too smooth, and the seasonal variation pattern may be distorted. $\Gamma$ is the local curve smoothness matrix consisting of scalars of the local smoothing parameter at all input data points [defined in (6)].

2) The value $d$ can be calculated in terms of the coefficients $b_{i}$ in matrix form with

$$
d=y-\mu \Gamma Q b^{\prime}
$$

and

$$
b^{\prime}=\left[\begin{array}{c}
b_{0} \\
b_{1} \\
b_{2} \\
\vdots \\
b_{n-1} \\
b_{n}
\end{array}\right]
$$

3) Finally, coefficients $a$ and $c$ are obtained from

$$
\begin{aligned}
& a_{i}=\frac{b_{i+1}-b_{i}}{3 h_{i}} \\
& c_{i}=\frac{d_{i+1}-d_{i}}{h_{i}}-\frac{h_{i}}{3}\left(b_{i+1}-2 b_{i}\right)
\end{aligned}
$$

for all $i=0,1, \ldots, n-1$.

Once the cubic splines $S_{i}(i=0,1, \ldots, n-1)$ are found, for the $i$ th adjacent time period $\left(x_{i}, x_{i+1}\right)$, the daily values of the surface parameter can be interpolated using $S_{i}$. After the coefficients $a, b, c$, and $d$ are calculated, the values of $y_{i}$ (e.g., LAI) at each $x_{i}$ (e.g., date of image acquisition) are estimated and denoted as $\hat{y}_{i}$. This procedure is iterated three or more times for each pixel. In each iteration, $y_{i}$ is replaced with $\hat{y}_{i}$ if $y_{i}<\hat{y}_{i}$.

This system of equations can be implemented in two ways.

1) GUCC. In this way, the global smoothing parameter is taken as a constant, ranging from 0.1 to 0.9 , and the curve smoothness matrix $\Gamma$ is given as a unity matrix.

2) LACC. In this case, the global smoothing parameter is set at 0.5 , but the matrix $\Gamma$ is defined as

$$
\Gamma=\left[\begin{array}{ccccc}
\gamma_{00} & 0 & \ldots & 0 & 0 \\
0 & \gamma_{11} & \ldots & 0 & 0 \\
\vdots & \vdots & \ddots & \vdots & \vdots \\
0 & 0 & \ldots & \gamma_{(n-1)(n-1)} & 0 \\
0 & 0 & \ldots & 0 & \gamma_{n n}
\end{array}\right]
$$

where $\gamma_{00}, \gamma_{11}, \ldots, \gamma_{n n}$ are calculated based on the local curvature (second derivative $y_{i}^{\prime \prime}$ at data point $i$ ) of the fitted curve by GUCC with $\lambda=0.5$, as follows:

$$
\gamma_{i i}=1-\left(\frac{\left|y_{i}^{\prime \prime}\right|}{y_{\max }^{\prime \prime}}\right)^{\frac{1}{2.5}}
$$

under the condition that if $\left|y_{i}^{\prime \prime}\right|>y_{\max }^{\prime \prime}$ then $\left|y_{i}^{\prime \prime}\right|=y_{\max }^{\prime \prime}$, where $y_{\max }^{\prime \prime}$ is the maximum second derivative found in the GUCC-fitted curve over the full year for a given pixel. The local curvature $y_{i}^{\prime \prime}$ can be either positive or negative. The positive value often occurs at the beginning of the growing season when the growth is accelerating, showing a concave shape of the LAI curve. It sometimes also occurs at the very end of the growing season when the LAI decreasing rate is decelerating, also making a concave curve shape. For other times of the year, the curve is generally either flat (i.e., $y_{i}^{\prime \prime} \approx 0$ ) before and after the growing season as well as in the midsummer or convex (i.e., $y_{i}^{\prime \prime}<0$ ) in either side of the midsummer plateau. As the absolute value of $y_{i}^{\prime \prime}$ is generally larger in the concave curve portion than in the convex curve portion, we only 
make use of the maximum positive value of $y_{i}^{\prime \prime}$. In this way, we emphasize the importance of capturing the rapid rise of LAI in the beginning of the growing season while avoiding using large negative curvatures caused by any abnormal convex curves. The $\gamma_{i i}$ value obtained in this way would range from 0 to 1 . At the point where the largest concave curvature occurs $\left(y_{\max }^{\prime \prime}\right), \gamma_{i i}=0$. As in (2), $\gamma_{i i}$ is multiplied with $\mu$, where $\mu=(2(1-\lambda) / 3 \lambda)$, $\gamma_{i i}=0$ is equivalent of $\lambda=1$, meaning no smoothing is applied so as to allow the fitted curve to be as close to the original data point as possible. Conversely, $\gamma_{i i}=1$ means that the local smoothing has the same strength as the GUCC, i.e., $\lambda=0.5$ in this study. The LACC method therefore effectively allows $\lambda$ to vary between 0.5 and 1 . The exponent $(1 / 2.5)$ is given a smaller value than unity not to make the $\lambda$ value overly sensitive to local curvature. GUCC is a simple method used here as compared with the advanced method of LACC. The advantages of LACC over GUCC are demonstrated in Section IV.

\section{Test Data AND PRocedure}

The GUCC and LACC methods are tested against an available LAI image series. Forty-six LAI maps in 2001 from the MODIS sensor (MOD15A2) over the entire China's landmass are processed to test the methods. The LAI of cloudcontaminated pixels labeled in MOD15A2 with "significant clouds were present" and "mixed clouds were present" are excluded from further analysis. For each pixel, the GUCC and LACC methods are used with "cloudless" LAI values to reconstruct the seasonal variation through identifying and replacing residual atmosphere-contaminated data points.

In applying these methods for image analysis, the input data include a time series of LAI, land cover, and the image acquisition date. Additional parameters such as the global smoothing parameter $\lambda$ and the number of total LAI scenes should also be defined. In a large geographical area such as China, there are often pixels with only a small number of cloud-free dates for which the trajectory analysis is not effective and a preprocessing procedure is needed. This procedure includes: 1) identifying pixels to be excluded from the trajectory analysis. These pixels include nonvegetated pixels and those with the total number of cloudless scenes fewer than 20 and 2) treatments for these excluded pixels. The nonvegetated pixels, which are labeled as urban and built-up, snow and ice, barren/sparsely, and water bodies, are assigned a zero LAI value. For any vegetated pixel with cloud-free dates fewer than 20, its LAI values are replaced with LAI values of a nearest pixel with same land cover type.

\section{RESUlts}

The large geographical area of China covers a wide range of ecosystems including subtropical forests in the south, temperate forests and grassland at midlatitudes, and boreal ecosystems in the north, with cropland interspersed within a large latitudinal range. These ecosystems have different seasonal variation patterns. Six pixels of different cover types across China are selected to demonstrate the effectiveness of the GUCC and



Fig. 1. Test pixel distribution in China in a MODIS land cover map. ENF evergreen needleleaf forest; $\mathrm{EBF}$, evergreen broadleaf forests; DNF, deciduous needleleaf forests; DBF, deciduous broadleaf forests; MXF, mixed forests; $\mathrm{CSH}$, closed shrublands; OSH, open shrublands; WSA, woody savannas; SAV, savannas; GRL, grasslands; PWL, permanent wetlands; CRL, croplands; URB, urban and built-up lands; CRM, cropland/natural vegetation mosaics; SNI, snow and ice; BSV, barren/sparse; WAT, water bodies.

TABLE I

Attributes of PiXels in 2001 Selected for Testing LACC and GUCC Methods. The Spatial Distribution of THESE PIXELS IS SHOWN IN FIG. 1

\begin{tabular}{|c|c|c|c|c|c}
\hline Name & Land Cover & Latitude(N) & Longitude(E) & $\begin{array}{c}\text { Maximum } \\
\text { LAI }\end{array}$ & $\begin{array}{c}\text { Number of } \\
\text { Clear } \\
\text { scenes }\end{array}$ \\
\hline CRL & Cropland & $30^{\circ} 55^{\prime} 55.3^{\prime \prime}$ & $119^{\circ} 54^{\prime} 17.2^{\prime \prime}$ & 6.6 & 40 \\
\hline DBF & $\begin{array}{c}\text { Deciduous } \\
\text { Broadleaf } \\
\text { Forest }\end{array}$ & $32^{\circ} 07^{\prime} 36.5^{\prime \prime}$ & $109^{\circ} 13^{\prime} 08.1^{\prime \prime}$ & 6.6 & 40 \\
\hline ENF & $\begin{array}{c}\text { Evergreen } \\
\text { Needleleaf } \\
\text { Forest }\end{array}$ & $25^{\circ} 44^{\prime} 14.3^{\prime \prime}$ & $117^{\circ} 01^{\prime} 19.6 ”$ & 6.6 & 33 \\
\hline GRL & $\begin{array}{c}\text { Grasslands } \\
\text { MXF }\end{array}$ & $33^{\circ} 13^{\prime} 13.2^{\prime \prime}$ & $102^{\circ} 28^{\prime} 21.1^{\prime \prime}$ & 6.6 & 43 \\
\hline PWL & $\begin{array}{c}\text { Permanent } \\
\text { Wetlands }\end{array}$ & $28^{\circ} 28^{\prime} 44.8^{\prime} "$ & $106^{\circ} 03^{\prime} 55.9^{\prime} "$ & 6.5 & 30 \\
\hline
\end{tabular}

LACC methods. The spatial distribution of these pixels is shown in Fig. 1 with a land cover map derived using MODIS data (EOS Data Gateway), and the attributes of these pixels are given in Table I.

The original MODIS "cloud-free" LAI time series of these pixels in 2001 are shown in Fig. 2(a)-(f). Also shown are a smooth capping curve fitted with the LACC method and those with the GUCC method using different global smoothing coefficients $(\lambda)$. From these examples, it is obvious that many cloudfree dates in MOD15A2 during the growing season produce erratic LAI values. These erratic variations in a short time were generally caused by the atmospheric effect or other artifacts rather than by the surface changes and can be detected with almost all $\lambda$ values smaller than 1. As in GUCC and LACC methods, these atmospherically contaminated data points are replaced with fitted data through an iteration procedure, the purpose of using these methods is to produce a seasonal trajectory that is the upper outline (capping) of all data points in the growing season. Large $\lambda$ values allow the fitted curves to 


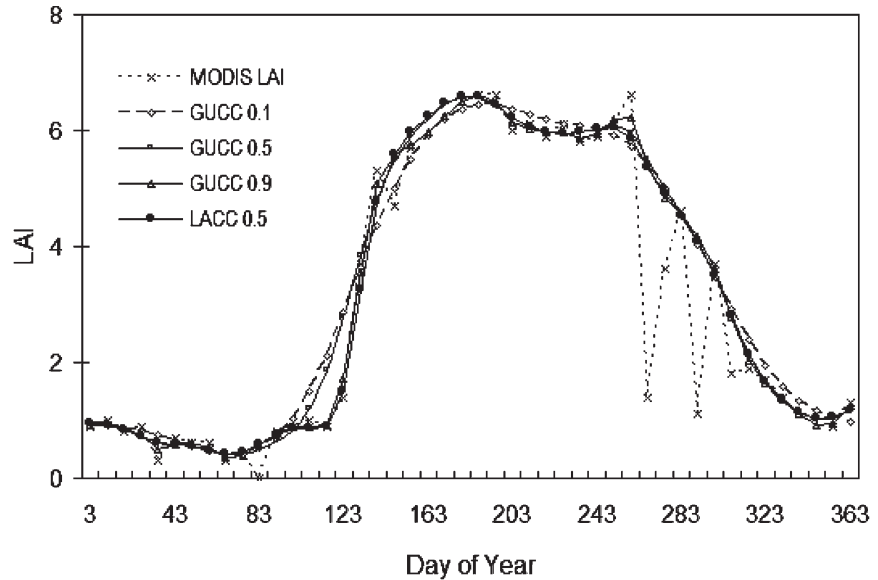

(a)

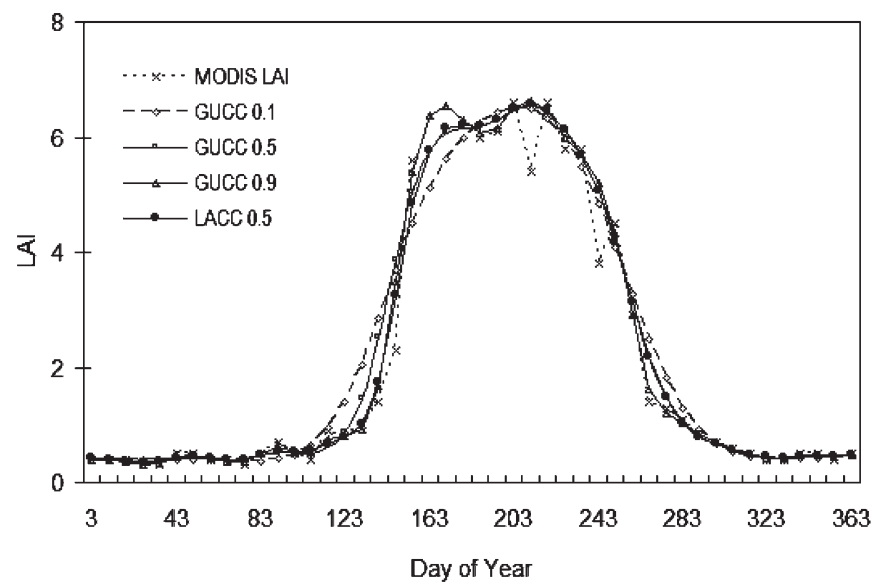

(c)

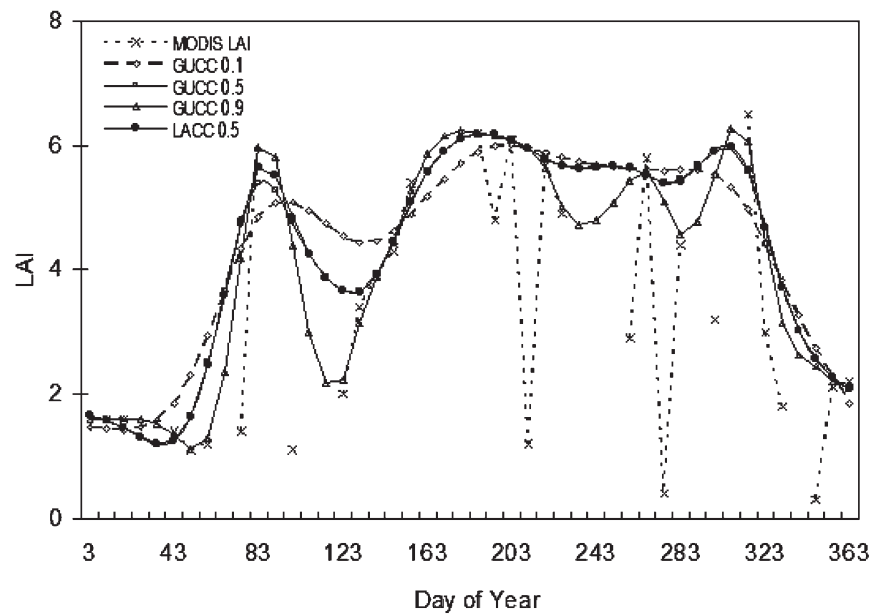

(e)

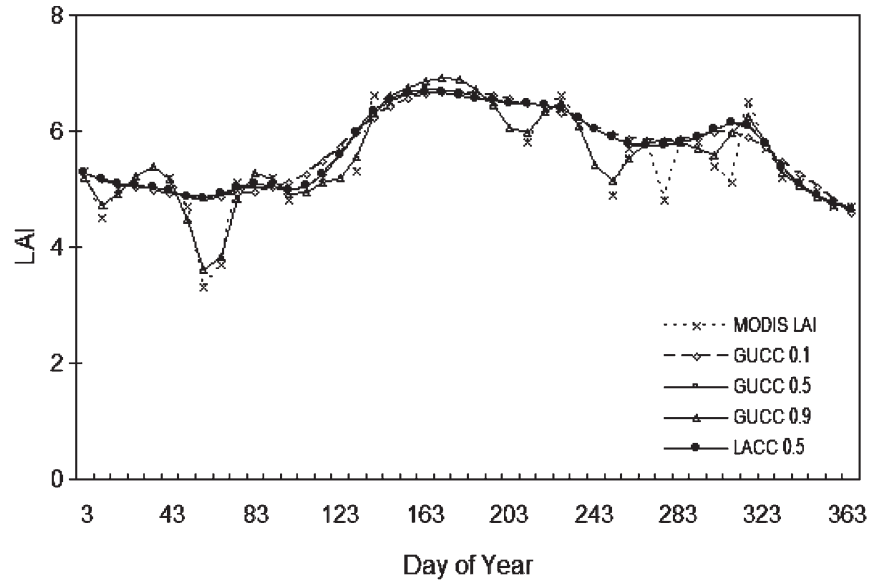

(b)

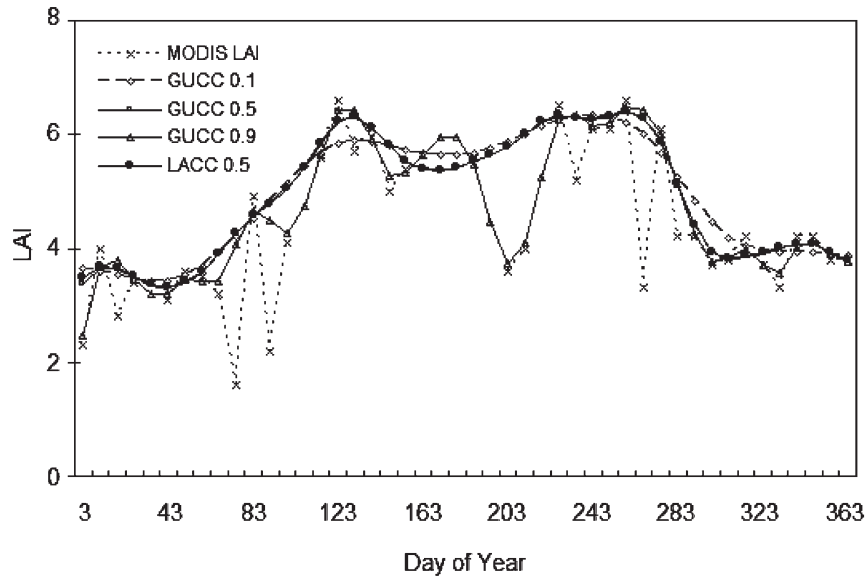

(d)

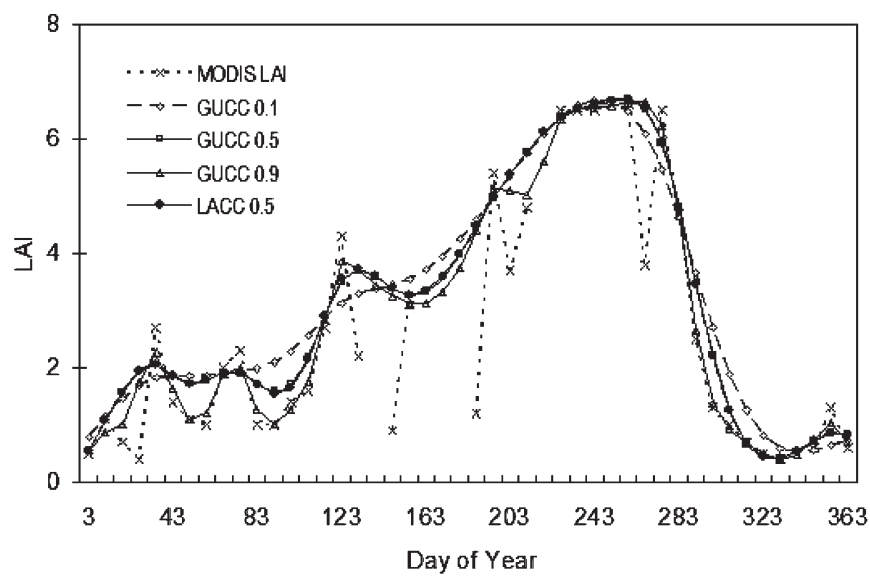

(f)

Fig. 2. Seasonal capping curves derived with GUCC with different values of the global smoothing parameter and with LACC. Test LAI data are from MOD15A2 labeled as cloud-free for different ecosystems in China (locations shown in Fig. 1). (a) Deciduous broadleaf forest. (b) Evergreen needleleaf forest. (c) Grassland. (d) Mixed forest. (e) Permanent wetland. (f) Cropland.

follow data points closely, but they are sometimes too flexible to be useful for detecting erratic data points. Smaller $\lambda$ values make it easier to find these erratic LAI values, but the fitted curves are generally too smooth to follow the rapid changes at the beginning and end of the growing season. For the broadleaf deciduous pixel [Fig. 2(a)], the curve with $\lambda=0.9$ captures the detailed variation patterns at the beginning and end of the grow- ing season, whereas curves with $\lambda=0.1$ and $\lambda=0.5$ are too smooth to show these patterns. The curve produced with LACC performs similarly to GUCC with $\lambda=0.9$. The LACC curve is smoother than that of GUCC in the middle of the growing season as it is less affected by the outstandingly large value at DOY $=55$, indicating some ability of the LACC method in suppressing isolated abnormally large LAI values. For an 
evergreen needleleaf pixel [Fig. 2(b)] with gentle variations throughout the year, GUCC with $\lambda=0.1$ and $\lambda=0.5$ produced similar results, and the resulting curves follow the general wavy features closely. The LACC method, which is based on $\lambda=0.5$, shows similar behaviors to GUCC with $\lambda=0.5$, whereas GUCC with $\lambda=0.9$ tracks almost every wiggle in the measured data except for a few small dips, defeating the purpose of finding a seasonal cap. In another similar case (not shown), GUCC with $\lambda=0.1$ generates uncharacteristic variation patterns, suggesting that this extreme value should not be used in general applications. The case of grassland [Fig. 2(c)] is similar to that of deciduous forest [Fig. 2(a)], where curves produced by LACC and GUCC with $\lambda=0.9$ follow the rapid changes at the beginning and end of the growing season. In this case, the GUCC curve at $\lambda=0.9$ is also unnecessarily more variable than the LACC curve in the middle of the growing season. GUCC curves with $\lambda=0.1$ and $\lambda=0.5$ are also too smooth to generate the near square-wave shape. For a mixed forest pixel with moderate seasonal variations but large erratic changes [Fig. 2(d)], the advantage of LACC over GUCC at $\lambda=0.9$ is clearly demonstrated: the former produces an ideal capping, whereas the latter is affine to several erratic changes, which are unnatural for forest ecosystems. In this case, GUCC with $\lambda=0.1$ is again too smooth, and GUCC with $\lambda=0.5$ produced similar results as LACC. In the case of a permanent wetland pixel [Fig. 2(e)], there is an uncharacteristically high $\mathrm{LAI}$ value at $\mathrm{DOY}=80$. It could be induced by a green flush or an unknown error. If this is an erroneous data point, GUCC and LACC are incapable of removing it, as the basic assumption used in these methods is that the atmospheric effect only causes negative biases in LAI. Under this circumstance, GUCC with $\lambda=0.9$ mimics the rapid changes, whereas LACC shows some inertia. Otherwise, LACC produces an effective capping curve. For a cropland pixel [Fig. 2(f)], several unusually large LAI values occurred before the peak growth, GUCC with $\lambda=0.9$ is again too variable, and GUCC with $\lambda=0.1$ is too smooth. LACC and GUCC with $\lambda=0.5$ behave similarly. They also effectively dampen several uncharacteristically large LAI values. This case also demonstrates that LACC is capable of simulating bimodal or multimodal seasonal variation patterns. From the above examples, we conclude that the GUCC method, which uses only a global smoothing parameter, is incapable of simulating all seasonal variation patterns, whereas the LACC method performs well under all circumstances tested. This is because LACC can automatically adjust the local smoothing parameter according to the local curvature of the curve first fitted with $\lambda=0.5$.

To assess quantitatively the ability of the LACC method in reconstructing an atmospherically contaminated time series of a surface parameter, we conducted a set of controlled numerical experiments. Fig. 3 shows an example of the experiments where an originally smooth LAI time series was artificially contaminated by randomly reducing $55 \%$ of the data points in the range from 0 to $100 \%$, and the contaminated series was then reconstructed using the LACC method. The reconstructed series resemble closely the originally smooth curve but differ by a small extent because of the large fraction of data contaminated and the limited three iterations. The reconstructed curve can be



Fig. 3. Example of reconstruction of a LAI time series using the LACC method. Fifty-five percent of the data points in an original LAI series were randomly reduced ("Disturbed") by $0-100 \%$, and the disturbed curve was then reconstructed using the LACC method.

made closer to the original data points if the number of iterations increases. Based on ten similar numerical experiments, we conclude that $92 \%$ of the artificial reduction in LAI can be recovered by the LACC method with three iterations. The recovery rate increased to $94 \%$ with ten iterations, suggesting that the LACC method may be used with more than three iterations for the best results when the speed of image processing is not a concern.

The effectiveness of the LACC method is further demonstrated by processing MODIS LAI images over the entire landmass of China. Example pairs of LAI images before and after the processing are shown in Fig. 4 . In the original MODIS LAI image on DOY 107 [Fig. 4(a)], southern China has large areas (including part of Taiwan) with $\mathrm{LAI}=0$, which are cloudaffected pixels. After LACC processing, these areas exhibit large LAI values filled mostly through temporal interpolation [Fig. 4(b)]. On DOY 221, the original image [Fig. 4(c)] has few cloud-affected pixels, but after LACC processing, there are obvious increases in LAI in several areas including southwest and northeast provinces [Fig. 4(d)]. These areas were mostly labeled as cloud-free, but the residual cloud/atmosphere effects were considerable. They are largely removed through LACC processing, i.e., these pixels with considerable negative biases in the LAI time series are identified and replaced with the values of the capping curves. On DOY 299, there is a large area in the southwest with LAI $=0$ in the original image [Fig. 4(e)]. After applying LACC, the LAI in this area increases significantly [Fig. 4(f)]. This is again mostly achieved through temporal interpolation with the capping curves. Statistically, the LACC processing increased the China-wide average LAI from 0.65 , 2.39 , and 0.79 to $0.97,2.98$, and 1.10 for DOY 107,211 , and 299, respectively. These examples demonstrate that: 1) residual atmospheric effects are considerable in the MODIS LAI time series and 2) these effects can be effectively removed or minimized by LACC.

In processing the images shown in Fig. 4, the whole year of data are needed to reconstruct a seasonal trajectory for each pixel to be used for replacing atmospherically affected dates through temporal interpolation. At a given date, the curvature of the trajectory can vary depending on the cover type and stage of vegetation development, resulting in different values of the local smoothing parameter in the LACC method. Fig. 5 shows 
(a)



(c)

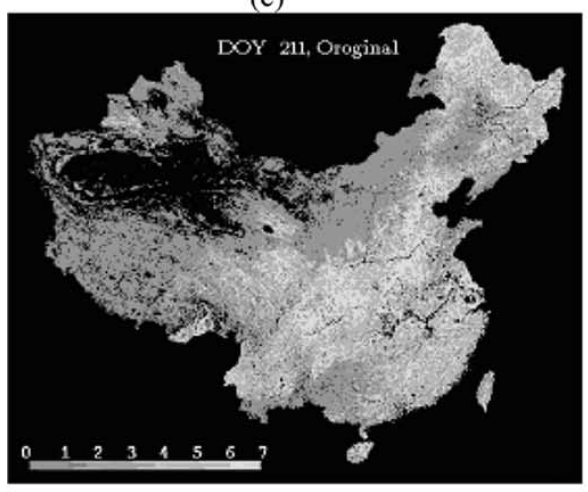

(e)

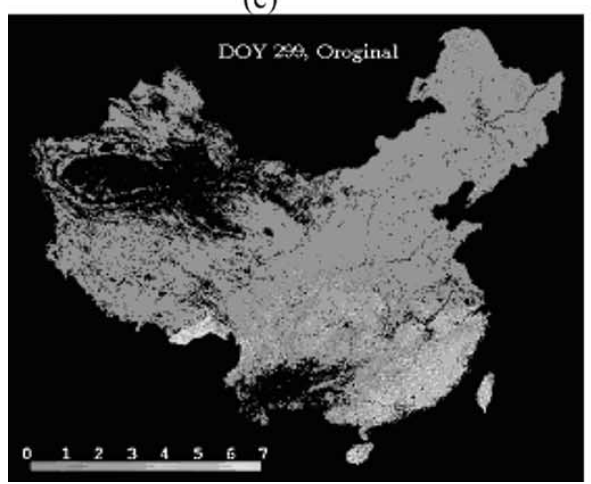

(b)



(d)



(f)

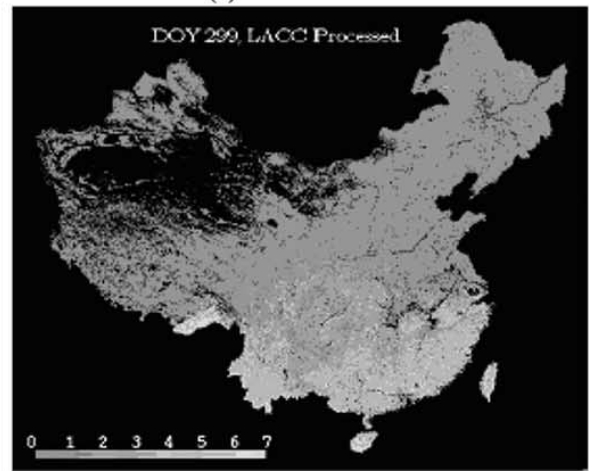

Fig. 4. Comparison of the original and LACC-processed LAI images of China on three composite dates (DOY) using the MODIS LAI product (MOD15A2) in 2001 .



Fig. 5. Histogram of the value of the local smoothness scaling parameter $\gamma_{i i}$ [used in (2) and defined in (7)] for May 2001 over China's landmass, where $\gamma_{i i}=0$ and $\gamma_{i i}=1$ are equivalent to the smoothing parameter $\lambda=1$ and $\lambda=0.5$, respectively, as the global smoothing parameter is set at 0.5 in LACC. a histogram of the scalar $\gamma_{i i}$ [defined in (7) and used in (2)] of this smoothing parameter in May, where $\gamma_{i i}=0$ corresponds to $\lambda=1$ and $\gamma_{i i}=1$ corresponds to $\lambda=0.5$. The value of $\gamma_{i i}$ varies in the full range from 0 to 1 . For pixels with small LAI temporal variations (small curvature) in May, the $\gamma_{i i}$ value is large, making the local $\lambda$ value small, but for pixels with large LAI changes (such as the beginning of vegetation growth), $\gamma_{i i}$ is small, making the local $\lambda$ value large. From the histogram, we see that $\gamma_{i i}$ has a peak value at about 0.45 , meaning that the most frequently used value of the local smoothing parameter $\lambda$ was $2 / 3$.

\section{CONClusion}

The LACC method developed in this study is shown to be effective in reconstructing seasonal trajectories of a surface parameter (LAI) through identifying and replacing 
atmosphercally affected data points by temporal interpolation. This method can be used as an additional quality control for temporal series of any surface parameters, which are negatively affected by inaccuracies in atmospheric corrections or by other artifacts. Compared with existing methods developed for the same purpose, LACC has the following characteristics.

1) It is flexible to fit any seasonal variation patterns. This ability of LACC is demonstrated for several cover types in China. This flexibility cannot be achieved using methods based on a small number of harmonics, which are theoretically inefficient in simulating rapid changes.

2) It fits variable curvatures of a surface parameter in all seasons through automatic adjustments of the local smoothing parameter. These adjustments are made based on the curvature of a preliminary curve fitted with a global smoothing parameter. This variable local smoothing parameter provides a smooth capping for seasons with slow changes in the surface parameter and, in the meantime, mimics rapid changes at the beginning and end of the growing season.

3) It produces a mathematically smooth capping curve throughout the year, which is differentiable and would be useful for determining growing season parameters.

It is suggested that seasonal trajectories be used as an additional quality control on surface parameters derived from moderate and coarse resolution images before they are used for further analysis and modeling.

\section{ACKNOWLEDGMENT}

S. Plummer and O. Arino of ESA provided useful comments on the early results. The $\mathrm{C}$ code for locally adjusted cubicspline capping is available on request.

\section{REFERENCES}

[1] J. M. Chen and T. A. Black, "Defining leaf area index for non-flat leaves," Plant Cell Environ., vol. 15, no. 4, pp. 421-429, 1992.

[2] J. M. Chen, G. Pavlic, L. Brown, J. Cihlar, S. G. Leblanc, H. P. White, R. J. Hall, D. Peddle, D. J. King, J. A. Trofymow, E. Swift, J. Van der Sanden, and P. Pellikka, "Validation of Canada-wide leaf area index maps using ground measurements and high and moderate resolution satellite imagery," Remote Sens. Environ., vol. 80, no. 1, pp. 165-184, Apr. 2002.

[3] J. Cihlar, J. M. Chen, and Z. Li, "Seasonal AVHRR composite multichannel data sets for scaling up," J. Geophys. Res., vol. 102, no. D24, pp. 29,625-29,640, 1997.

[4] J. Cihlar, H. Ly, Z Li, J. M. Chen, H. Pokrant, and F. Huang, "Multitemporal, multichannel AVHRR data sets for land biosphere studies: Artifacts and corrections," Remote Sens. Environ., vol. 60, no. 1, pp. 35-57, Apr. 1997.

[5] F. Cipriani and E. Cubero-Castan, "Intermediate scale approach for estimating vegetation canopy leaf area index using SPOT4/VGT spectral bands," in Proc. VEGETATION, Lake Maggiore, Italy, 2000.

[6] W. S. Cleveland and S. J. Devlin, "Locally weighted regression: An approach to regression analysis by local fitting," J. Amer. Statist. Assoc., vol. 83, no. 403, pp. 596-608, 1988.

[7] A. Henderson-Sellers, "Defogging cloud determination algorithms," Nature, vol. 298, no. 5873, pp. 419-420, 1982.

[8] J. Hu, B. Tan, N. Shabanov, K. A. Crean, J. V. Martonchik, and D. J. Diner, "Performance of the MISR LAI and FPAR algorithm: A case study in Africa," Remote Sens. Environ., vol. 88, no. 3, pp. 324-340, Dec. 2003.

[9] S. Liang, H. Fang, M. Chen, C. J. Shuey, C. Walthall, C. Daughtry, J. Morisette, C. Schaaf, and A. Strahler, "Validating MODIS land surface reflectance and albedo products: Methods and preliminary results," Remote Sens. Environ., vol. 83, no. 1/2, pp. 149-162, Nov. 2002
[10] A. Lotsch, Y. Tian, M. A. Friedl, and R. B. Myneni, "Land cover mapping in support of LAI and FPAR retrievals from EOS-MODIS and MISR: Classification methods and sensitivities to errors," J. Amer. Statist. Assoc., vol. 24, no. 10, pp. 1997-2016, May 2003.

[11] R. B. Myneni, S. Hoffman, Y. Knyazikhin, J. L. Privette, J. Glassy, Y. Tian, Y. Wang, X. Song, Y. Zhang, G. R. Smith, A. Lotsch, M. Friedl, J. T. Morisette, P. Votava, R. R. Nemani, and S. W. Running, "Global products of vegetation leaf area and fraction absorbed PAR from year one of MODIS data," Remote Sens. Environ., vol. 83, no. 1/2, pp. 214-231, Nov. 2002.

[12] C. L. Parkinson, EOS Data Products Handbook, vol. 2. Greenbelt, MD NASA/Goddard Space Flight Center, 2000.

[13] S. Plummer, J. M. Chen, G. Dedieu, and M. Simpson, GLOBCARBON Expert Support Laboratory Input and Output Definition, 2002, Frascati, Italy: ESRIN, European Space Agency. GLBC/ESL/IODD version 1.0.

[14] D. S. G. Pollock, A Handbook of Time-Series Analysis, Signal Processing and Dynamics. New York: Academic, 1999.

[15] G. J. Roerink, M. Menenti, and W. Verhoef, "Reconstructing cloud free NDVI composites using Fourier analysis of time series," Int. J. Remote Sens., vol. 21, no. 9, pp. 1911-1917, Jun. 2000.

[16] P. J. Sellers, S. O. Los, C. J. Tucker, C. O. Justice, D. A. Dazlich, G. J. Collatz, and D. A. Randall, "A global $1 * 1$ degree NDVI data set for climate studies. Part 2: The generation of global fields of terrestrial biophysical parameters from the NDVI," Int. J. Remote Sens., vol. 15, no. 17, pp. 3519-3545, 1994

[17] J. J. Simpson and J. I. Gobat, "Improved cloud detection for daytime AVHRR scenes over land," Remote Sens. Environ., vol. 55, no. 1, pp. 21-49, Jan. 1996

[18] L. L. Stowe, E. P. McClain, R. Carey, P. Pellegrino, G. G. Gutman, P. Davis, C. Long, and S. Hart, "Global distribution of cloud cover derived from NOAA/AVHRR operational satellite data," Adv. Space Res., vol. 11, no. 3, pp. 51-54, 1991.

[19] J. J. Tuma, Engineering Mathematics Handbook. New York: McGraw-Hill, 1998.

[20] W. Verhoef, M. Menenti, and S. Azzali, "A colour composite of NOAAAVHRR-NDVI based on times series (1981-1992)," Int. J. Remote Sens., vol. 17 , no. 2, pp. 231-235, 1996.

[21] E. F. Vermote, N. El Saleous, C. O. Justice, Y. J. Kaufman, J. L. Privette, L. Remer, J. C. Roger, and D. Tanre, "Atmospheric correction of visible to middle-infrared EOS-MODIS data over land surfaces: Background, operational algorithm and validation," J. Geophys. Res.-Atmos., vol. 102, no. D14, pp. 17131-17141, Jul. 1997.

[22] E. F. Vermote, N. El Saleous, and C. O. Justice, "Atmospheric correction of MODIS data in the visible to middle infrared: First results," Remote Sens. Environ., vol. 83, no. 1/2, pp. 97-111, Nov. 2002.

[23] N. Viovy and O. Arino, "The best index slope extraction (BISE): A method for reducing noise in NDVI time-series," Int. J. Remote Sens., vol. 13 , no. 8 , pp. $1585-1590,1992$.

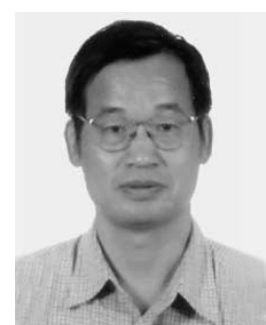

Jing M. Chen received the B.Sc. degree in applied meteorology from the Nanjing Institute of Meteorology, Nanjing, China, in 1982, and the Ph.D. degree in meteorology from Reading University, Reading, U.K., in 1986.

From 1989 to 1993, he was a Postdoctoral Fellow and Research Associate at the University of British Columbia, Vancouver, BC, Canada. From 1993 to 2000, he was a Research Scientist at the Canada Centre for Remote Sensing, Ottawa, ON. He is currently a Professor and a Canada Research Chair at the Department of Geography, University of Toronto, Toronto, ON, and an Adjunct Professor at York University, Toronto. His recent research interests are in the remote sensing of biophysical parameters, plant canopy radiation modeling, terrestrial water and carbon cycle modeling, and atmospheric inverse modeling for global and regional carbon budget estimation. He has published over 120 papers in refereed journals.

Dr. Chen served as an Associate Editor of the IEEE TRANSACTIONS ON Geoscience AND ReMOTE SENSING from 1996 to 2002. 


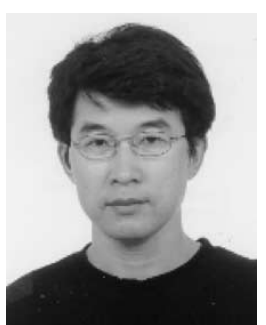

Feng Deng received the B.S. degree in 1985 and M.S. degree in geography and climatology in 1988 from the East China Normal University, Shanghai, China.

He was a Researcher at the Institute of Environmental Studies of Ningbo, China. He is currently with the Department of Geography and Planning Program, University of Toronto, Toronto, ON, Canada. His research interests are the application of remote sensing and geographic information system in the field of global changes.

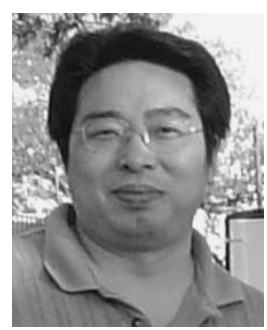

Mingzhen Chen received the Ph.D. degree in remote sensing of soils from Zhejiang University, Zhejiang, China in 1996.

From 1996 to 1998, he was an Associate Professor at Zhejiang University. From 1999 to 2001, he was a Visiting Research Scientist at the University of Maryland, College Park. He was a Research Associate previously at the University of Toronto and currently at Auburn University, Auburn, AL. His research interests include remote sensing and geographic information system (GIS) applications, such as retrieving land surface parameters from remotely sensed data, land cover and land use, digital image processing, natural resources inventory and management, biomass and environmental monitoring, and GIS system design and implementation. 